\title{
Construction and test of a moving boundary model for negative streamer discharges
}

\author{
Fabian Brau, ${ }^{1}$ Alejandro Luque, ${ }^{1}$ Bernard Meulenbroek,,${ }^{1,2}$ Ute Ebert, ${ }^{1,3}$ and Lothar Schäfer ${ }^{4}$ \\ ${ }^{1}$ Centrum voor Wiskunde en Informatica (CWI), P. O. Box 94079, 1090 GB Amsterdam, Netherlands \\ ${ }^{2}$ Faculty of Electrical Engineering, Mathematics and Computer Science, Delft University of Technology, Delft, The Netherlands \\ ${ }^{3}$ Department of Physics, Eindhoven University of Technology, Eindhoven, The Netherlands \\ ${ }^{4}$ Department of Physics, Universität Essen-Duisburg, Duisburg, Germany
}

(Received 7 July 2007; published 28 February 2008)

\begin{abstract}
Starting from the minimal model for the electrically interacting densities of electrons and ions in negative streamer discharges, we derive a moving boundary approximation for the ionization fronts. Solutions of the moving boundary model have already been discussed, but the derivation of the model was postponed to the present paper. The key ingredient of the model is the boundary condition on the moving front. It is found to be of kinetic undercooling type, and the relation to other moving boundary models is discussed. Furthermore, the model is compared to two-dimensional simulations of the underlying density model. The results suggest that our moving boundary approximation adequately represents the essential dynamics of negative streamer fronts.
\end{abstract}

DOI: $10.1103 /$ PhysRevE.77.026219

PACS number(s): 89.75.Kd, 47.54.Bd, 52.80.-s, 82.40.Ck

\section{INTRODUCTION}

Streamers appear when a sufficiently strong voltage is applied to an ionizable gas, liquid, or solid with a low initial conductivity; they are growing plasma channels that expand by an ionization reaction. Streamers determine the initial stages of electric breakdown, sparks, and lightning and occur equally in technical and natural processes [1-4]. Negative (anode-directed) streamers in pure gases, such as nitrogen or argon, can be described on a mesoscopic level by a minimal streamer model of two reaction advection diffusion equations for the densities of electrons and positive ions, respectively, coupled to the electric field. Numerical solutions [5-13] of this minimal model reveal that after some initial avalanche phase [11], a streamer channel develops that is characterized by a thin space charge layer around its tip. This layer, where the electron density largely exceeds the density of positive ions, screens the electric field from the streamer interior and enhances it ahead of the streamer tip, creating there a high field zone where the ionization grows rapidly through an efficient impact ionization by field accelerated electrons. This self-generated field enhancement allows the streamer to penetrate areas where the background field is too low for efficient ionization. Figure 1 illustrates the development of this space charge layer, starting from a smooth initial ionization seed. More detailed illustrations of fully developed streamer fronts will be presented in Sec. IV. In the fully developed streamer, the width of the space charge layer is much smaller than the radius of the streamer head; this separation of scales is actually necessary for the strong field enhancement ahead of the streamer and the field screening from the ionized interior. It suggests a moving boundary approximation for the ionization front which brings the problem into the form of a Laplacian interfacial growth model. Such a model was first formulated by Lozansky and Firsov [14] and the concept was further detailed in $[9,15,16]$; solutions of such a moving boundary approximation were discussed in [17-20].

In the moving boundary approximation presented in $[14,17-20]$ the space charge layer, which is much thinner than its radius of curvature, is replaced by an infinitesimally thin interface on the scale of the outer regions. It is assumed that the streamer moves into a nonionized and electrically

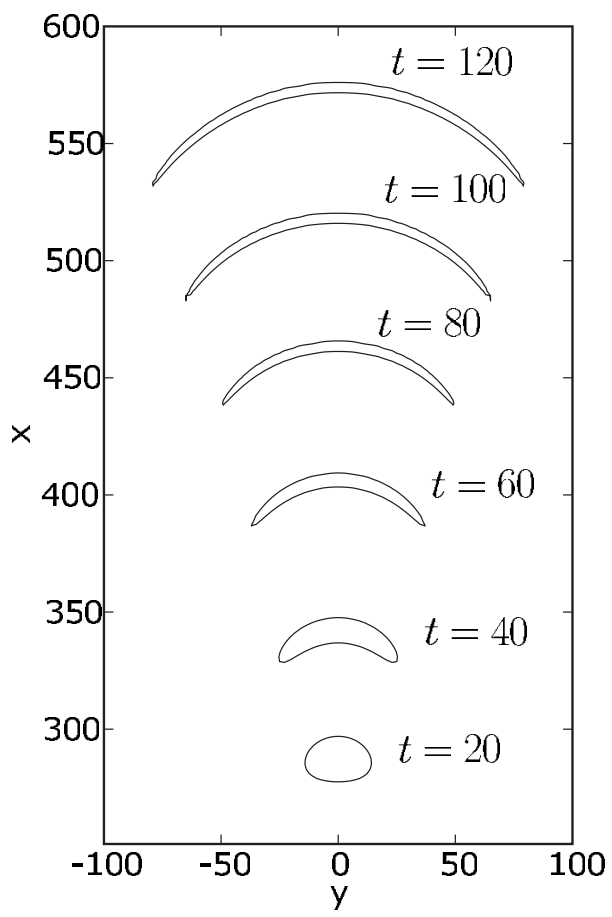

FIG. 1. Stages of evolution of the space charge layer surrounding a streamer in a homogeneous background electric field of $E_{0}$ $=1$ pointing downward. The full simulation are is $0 \leqslant x \leqslant 2048$ and $-1024 \leqslant y \leqslant 1024$, and only the relevant part is shown in the figure. Plotted is the half-maximum line of the negative space charge density $\sigma-\rho$ at instances of time $t$ as indicated. The model is the PDE streamer model (12)-(14) with $D=0.1$. Similar plots in three spatial dimensions with radial symmetry were presented and discussed in $[10,11,13]$ and complemented by plots of electron and ion densities in the streamer interior and of electric fields; the present simulation is purely two dimensional. 
neutral medium, so that outside the streamer the electric potential $\varphi$ obeys the Laplace equation

$$
\Delta \varphi=0 \quad \text { outside the streamer. }
$$

The ionized body of the streamer is modeled as ideally conducting,

$$
\varphi=\text { const inside the streamer. }
$$

We immediately note that this latter assumption will not be essential for our analysis. The interface separating these two regions moves with a velocity $v_{n}$ that depends on the local electric field

$$
v_{n}=v^{*}(\nabla \varphi)^{+} .
$$

Here and below, superscripts \pm indicate the limit value on the interface where the interface is approached from the outside or the inside, respectively.

In earlier work $[14,17]$, the electric potential was taken as continuous on the streamer boundary, $\varphi^{+}=\varphi^{-}$, which for an ideally conducting streamer interior implies that the moving interface is equipotential, $\varphi^{+}=$const. However, due to the small but finite width of the physical space charge layer this assumption is unfounded. Rather, in moving boundary approximation, $\varphi$ must be discontinuous at the interface. In $[18,19]$ we used

$$
\varphi^{+}-\varphi^{-}=\ell \hat{\mathbf{n}} \cdot(\nabla \varphi)^{+}
$$

to model the potential difference across the interface without discussing its derivation; here the length parameter $\ell$ accounts for the thickness of the ionization front and $\hat{\mathbf{n}}$ is the exterior normal on the interface. Derivation and discussion of this boundary condition (in Sec. III) and its test on numerical solutions of the density model (in Sec. IV) are the subject of the present paper. In the context of crystal growth from undercooled melts such a boundary condition is known as kinetic undercooling.

Clearly the model (1)-(4) is intimately related to moving boundary models for a variety of different physical phenomena, such as viscous fingering (see, e.g., $[21,22]$ ) or crystal growth (see, e.g., $[23,24]$ ). To derive the model in the context of streamer motion, our starting point is the minimal streamer model that applies to anode-directed discharges in simple gases, such as nitrogen or argon. Cathode-directed discharges or discharges in composite gases such as air involve additional physical mechanisms [3].

Section II summarizes previous results as far as needed: The minimal streamer model in Sec. II A, and the properties of planar shock fronts with vanishing electron diffusion that move with constant velocity in an externally applied, timeindependent electric field in Sec. II B. Based on these results, in Sec. III A, supplemented by an appendix, we present a rigorous derivation of the boundary condition (4), valid for planar fronts in strong electric fields. The relation of our model to other moving boundary models is briefly discussed in Sec. III B. The crucial question whether the model also applies to curved ionization fronts with electron diffusion in weaker external fields is considered in Sec. IV. Based on numerical solutions of the minimal model in twodimensional space we argue that our moving boundary model indeed captures the essential physics of fully developed (negative) streamer fronts. Our conclusions are summarized in Sec. V.

\section{COLLECTION OF SOME PREVIOUS RESULTS}

\section{A. The minimal streamer model}

The model for negative streamers in simple nonattaching gases, such as pure nitrogen or argon, consists of a set of three coupled partial differential equations (PDEs) for the electron density $n_{e}$, the ion density $n_{+}$, and the electric field $\overline{\mathbf{E}}$,

$$
\begin{gathered}
\partial_{\bar{t}} n_{e}-\overline{\boldsymbol{\nabla}} \cdot\left(n_{e} \mu_{e} \overline{\mathbf{E}}+D_{e} \bar{\nabla} n_{e}\right)=\left|n_{e} \mu_{e} \overline{\mathbf{E}}\right| \bar{\alpha}(|\overline{\mathbf{E}}|), \\
\partial_{\bar{t}} n_{+}=\left|n_{e} \mu_{e} \overline{\mathbf{E}}\right| \bar{\alpha}(|\overline{\mathbf{E}}|), \\
\overline{\boldsymbol{\nabla}} \cdot \overline{\mathbf{E}}=\frac{e\left(n_{+}-n_{e}\right)}{\epsilon_{0}} .
\end{gathered}
$$

The first two equations are the continuity equations for the electrons and the ions while the last is the Coulomb equation for the electric field generated by the space charge $e\left(n_{+}\right.$ $-n_{e}$ ) of electrons and ions. The overbar denotes dimensional variables, $\mu_{e}$ and $D_{e}$ are mobility and diffusion constants of the electrons in the specific medium at given temperature and pressure, $e$ is the elementary charge, and $\epsilon_{0}$ is the dielectric constant of the vacuum (to be multiplied by the dielectric constant $\epsilon>1$ in dense media, such as liquids and solids). As the streamer evolves on the fast time scale of electron mobility, the ion motion is neglected. $\left|n_{e} \mu_{e} \overline{\mathbf{E}}\right| \bar{\alpha}(|\overline{\mathbf{E}}|)$ is the generation rate of additional electron ion pairs; it is the product of the absolute value of the drift current times the effective cross section

$$
\bar{\alpha}(|\overline{\mathbf{E}}|)=\alpha_{0} \alpha\left(|\overline{\mathbf{E}}| / E_{T}\right),
$$

which is taken as field dependent; an old and much used form is the Townsend approximation

$$
\alpha\left(|\overline{\mathbf{E}}| / E_{T}\right)=e^{-E_{T^{\prime}}|\overline{\mathbf{E}}|} .
$$

The parameters $\alpha_{0}$ and $E_{T}$ are specific for the ionizable medium, such as $\mu_{e}$ and $D_{e}$; they can vary by many orders of magnitude and typically scale with the density of the medium $[4,10,13]$. The validity of this model as an approximation for the full stochastic motion of single electrons and ions between abundant neutral molecules in nitrogen is discussed in $[26]$.

When rewriting the equations in dimensionless units as in many previous papers $[4,9,10,13,15,16]$, the ionization length in a strong electric field $1 / \alpha_{0}$ is taken as length scale and $E_{T}$ as the scale of the electric field. The characteristic scale of velocity is $\mu_{e} E_{T}$, and therefore the scale of time is $1 /\left(\alpha_{0} \mu_{e} E_{T}\right)$. Finally, the nondimensionalization of the Poisson equation shows that $\epsilon_{0} \alpha_{0} E_{T} / e$ is the characteristic scale for the electron and ion density. i.e., these densities will create fields of order $E_{T}$ on the length scale $1 / \alpha_{0}$. In the dimensionless units 


$$
\begin{gathered}
\mathbf{r}=\alpha_{0} \overline{\mathbf{r}}, \quad t=\frac{\bar{t}}{\alpha_{0} \mu_{e} E_{T}}, \quad D=\frac{D_{e} \alpha_{0}}{\mu_{e} E_{T}}, \\
\sigma=\frac{e n_{e}}{\alpha_{0} \epsilon_{0} E_{T}}, \quad \rho=\frac{e n_{+}}{\alpha_{0} \epsilon_{0} E_{T}}, \quad \mathbf{E}=\frac{\overline{\mathbf{E}}}{E_{T}},
\end{gathered}
$$

the model (5)-(7) reads as

$$
\begin{gathered}
\partial_{t} \sigma-\nabla \cdot(\sigma \mathbf{E}+D \nabla \sigma)=\sigma|\mathbf{E}| \alpha(|\mathbf{E}|), \\
\partial_{t} \rho=\sigma|\mathbf{E}| \alpha(|\mathbf{E}|), \\
\nabla \cdot \mathbf{E}=\rho-\sigma, \quad \mathbf{E}=-\nabla \varphi .
\end{gathered}
$$

Here the electrostatic approximation for the electric potential $\varphi$ was introduced which is appropriate for streamers [16]. The dimensionless diffusion constant $D$ is typically small and independent of the density of the medium; e.g., for nitrogen at room temperature, it is $D=0.1$ [16].

Finally, the effective impact ionization cross section is in Townsend approximation (9) given by $\alpha=e^{-1 /|\mathbf{E}|}$, but we will allow the more general form

$$
\begin{gathered}
\alpha(0)=0, \quad \frac{d \alpha(|\mathbf{E}|)}{d|\mathbf{E}|} \geq 0, \\
\alpha(|\mathbf{E}|) \underset{|\mathbf{E}| \gg 1}{=} 1-O\left(\frac{1}{|\mathbf{E}|}\right) .
\end{gathered}
$$

\section{B. Planar ionization fronts}

As is discussed in detail in Sec. 2 of [25], diffusion contributes little to the current in strong fields. Therefore, we here use the approximation $D=0$, and we will discuss this approximation further in Sec. IV of the present paper.

We here recall essential properties of planar negative ionization fronts for $D=0$ as derived in $[16,27]$. We consider ionization fronts propagating in the positive $x$ direction into a medium that is completely nonionized beyond a certain point $x_{f}(t)$,

$$
\begin{gathered}
\sigma=0=\rho \text { for } x>x_{f}(t), \\
\sigma>0 \text { for } x<x_{f}(t) .
\end{gathered}
$$

Far ahead of the front, the electric field is taken to approach a constant value,

$$
\underset{x \rightarrow+\infty}{\mathbf{E}}-E_{0} \hat{\mathbf{x}}, \quad E_{0}>0
$$

where $\hat{\mathbf{x}}$ is the unit vector in the $x$ direction. For a planar front, $\mathbf{E}$ evidently can depend only on $x$ and $t$, and Eqs. (14) and (17) yield a constant field in the nonionized region,

$$
\mathbf{E}=-E_{0} \hat{\mathbf{x}}, \quad x \geq x_{f} .
$$

The planar solution of the model takes the form of a uniformly translating shock front moving with velocity

$$
v=\partial_{t} x_{f}=-\left.E\right|_{x=x_{f}}=E_{0} .
$$

In the comoving coordinate

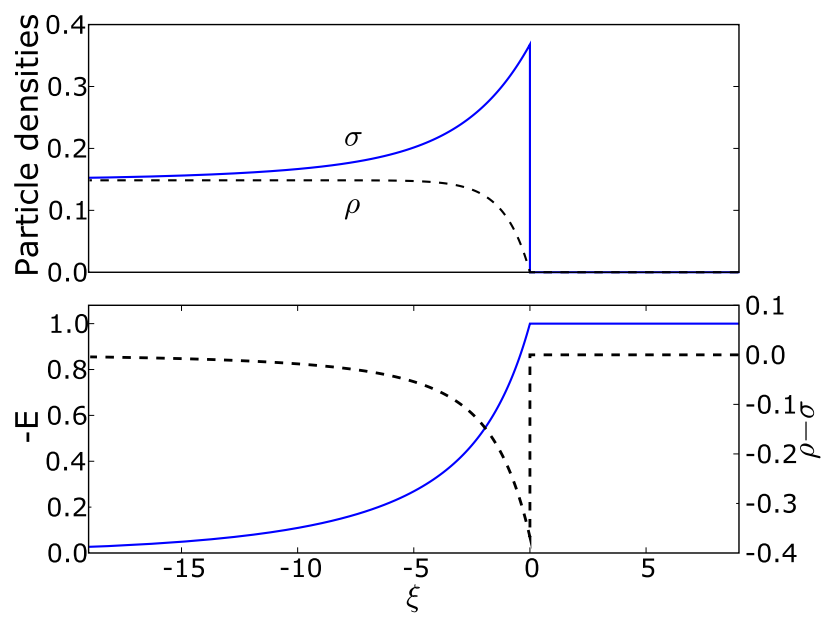

FIG. 2. (Color online) Densities and field in a planar ionization front in a far field $E_{0}=1$ for $D=0$ as a function of the comoving coordinate $\xi(20)$. The front moves to the right-hand side with velocity $v=E_{0}$. Upper panel, electron density $\sigma$ (solid line) and ion density $\rho$ (dashed line); lower panel, electric field (solid line, axis on the left-hand side) and space charge density $\rho-\sigma$ (dashed line, axis on the right-hand side). For $\alpha(|\mathbf{E}|)$ the Townsend approximation (9) was used.

$$
\xi=x-x_{f}(0)-v t
$$

a discontinuity of the electron density is located at $\xi=0$, while the ion density $\rho$ and the electric field $E$ are continuous.

Figure 2, which similarly has appeared in [27], illustrates the spatial profiles in such a uniformly translating front for $E_{0}=1$. In the nonionized region $\xi>0$, we simply have $\rho=0$ $=\sigma$ and $E=-E_{0}$. In the ionized region $\xi<0$, the propagating electron front creates additional electrons and ions as long as $\alpha(E)>0$, therefore the density of immobile ions $\rho$ increases monotonically behind the front. The electrons move such as to screen the conducting interior from the applied electric field. They form a layer of nonvanishing space charge $\rho-\sigma$ that suppresses the field $E$ behind the front.

Analytically the solution in the ionized region $\xi<0$ is given implicitly by the equations

$$
\begin{gathered}
\sigma(E)=\frac{E_{0}}{E_{0}-|E|} \rho(E), \\
\rho(E)=\int_{|E|}^{E_{0}} \alpha(\eta) d \eta, \\
-\xi=\int_{E(\xi)}^{-E_{0}} \frac{E_{0}+\eta}{\rho(\eta)} \frac{d \eta}{\eta} .
\end{gathered}
$$

In the limit $E_{0} \gg 1$, these equations will be further evaluated below. We note that at the shock front, the electron density jumps from zero to

$$
\sigma=E_{0} \alpha\left(E_{0}\right),
$$

and for $\xi \rightarrow-\infty$ it approaches the value 


$$
\sigma(-\infty)=\int_{0}^{E_{0}} \alpha(\eta) d \eta
$$

Far behind the front, where $\mathbf{E}$ is so small that $\alpha(|\mathbf{E}|) \simeq 0$, the final relaxation of $\mathbf{E}$ and of $\sigma$ is exponential in space, $\sim e^{-\lambda \xi}$, where

$$
\lambda=\frac{\sigma(-\infty)}{v}=\frac{1}{E_{0}} \int_{0}^{E_{0}} \alpha(\eta) d \eta \stackrel{E_{0} \rightarrow \infty}{\rightarrow} 1 .
$$

\section{MOVING BOUNDARY MODEL}

\section{A. Construction}

The results reviewed in the preceding section (see also Fig. 1) show that a layer of space charge $\rho-\sigma$ screens the electric field from the streamer interior. For strong applied electric field $-E_{0}$, the thickness of this layer defines some small inner scale of the front, while on the large outer scale, the streamer will be approximated as a sharp interface separating an ionized but electrically neutral region inside the streamer from the nonionized charge-free region outside the streamer; this substantiates the assumptions underlying the moving boundary model treated in $[18,19]$.

Being a shock front solution of Eq. (12), the interface always moves with normal velocity

$$
v_{n}=\hat{\mathbf{n}} \cdot(\nabla \varphi)^{+}
$$

where $\hat{\mathbf{n}}$ is the unit vector normal to the interface pointing into the exterior region; this equation generalizes Eq. (19). We recall that $\mathrm{a} \pm$ indicates that the expression is evaluated by approaching the interface from outside the streamer.

As mentioned in the Introduction, in the context of electric breakdown the moving boundary model (1), (2), and (27) has been formulated some time ago by Lozansky and Firsov [14]. To complete the model, a boundary condition at the interface is needed, and in Ref. [14] continuity of the potential at the interface was postulated,

$$
\varphi^{+}=\varphi^{-}=\text {const }
$$

However, Fig. 2 clearly shows that crossing the screening layer, $\varphi(\xi)$ will increase, which amounts to a jump $\varphi^{+}>\varphi^{-}$in the interface model. The size of this jump depends on the electric field $\mathbf{E}^{+}$at the interface and for a planar front is easily determined from Eq. (23). We note that in the framework of the PDE model (Sec. II), $\varphi^{ \pm}$are to be identified as

$$
\begin{gathered}
\varphi^{-}=\varphi(-\infty), \quad \varphi^{+}=\varphi(0), \\
\varphi^{+}-\varphi^{-}=-\int_{-\infty}^{0} E d \xi .
\end{gathered}
$$

Since $E(\xi)$ according to (22) and (23) is a monotonically decreasing function for $\xi<0$, we can integrate by parts

$$
\varphi^{+}-\varphi^{-}=-\int_{-\infty}^{0} E d \xi=\left.(\xi E)\right|_{-\infty} ^{0}+\int_{0}^{E^{+}} \xi d E=\int_{0}^{E^{+}} \xi d E .
$$

The last identity holds since either $E$ or $\xi$ vanishes in the integration boundaries. For a planar front, we have $E^{+}=-E_{0}$, but we here keep $E^{+}$to stress the dependence on the field at the front position $\xi=0$.

While $E(\xi)$ is known only implicitly as $\xi=\xi(E)$ in Eq. (23), the partial integration now allows us to evaluate the integral explicitly by substituting Eq. (23) in (31),

$$
\begin{aligned}
\varphi^{+}-\varphi^{-} & =-\int_{0}^{E^{+}} d E \int_{E}^{E^{+}} \frac{\eta-E^{+}}{\rho(\eta)} \frac{d \eta}{\eta} \\
& =-\int_{0}^{E^{+}} \frac{\eta-E^{+}}{\rho(\eta)} d \eta .
\end{aligned}
$$

$\rho(\eta)$ is given in Eq. (22). The result (33) is exact for uniformly translating planar fronts with vanishing diffusion; it explicitly shows that in the interface model the potential is discontinuous across the boundary, where the size of the discontinuity depends on the electric field right ahead of the ionization front.

Evaluating Eq. (33), in the Appendix we derive bounds showing that $\varphi^{+}-\varphi^{-} \approx-E^{+}+$const for large $\left|E^{+}\right|$. We present a simpler argument yielding only the leading term. It is based on direct evaluation of Eqs. (22) and (23), written as

$$
\begin{aligned}
& \frac{\rho(E)}{\left|E^{+}\right|}=\int_{E / E^{+}}^{1} \alpha\left(\eta\left|E^{+}\right|\right) d \eta, \\
& \xi=\left|E^{+}\right| \int_{E(\xi) / E^{+}}^{1} \frac{\nu-1}{\rho\left(\nu E^{+}\right)} \frac{d \nu}{\nu} .
\end{aligned}
$$

We now take the limit $\left|E^{+}\right| \rightarrow \infty$ in Eq. (34), with $E / E^{+}>0$ fixed. The asymptotic behavior (15) of $\alpha(|\mathbf{E}|)$ yields

$$
\frac{\rho(E)}{\left|E^{+}\right|}=1-\frac{E}{E^{+}} .
$$

Substituting this result into Eq. (35) we find

$$
\xi=-\int_{E(\xi) / E^{+}}^{1} \frac{d \nu}{\nu}
$$

yielding a purely exponential front profile

$$
E(\xi)=E^{+} e^{\xi} .
$$

This result means that the exponential decay of the space charge layer (26), which holds far behind the front for all $\left|E^{+}\right|>0$, for $\left|E^{+}\right| \gg 1$ is actually valid throughout the complete front up to $\xi=0$. Substituting Eq. (37) into Eq. (30), we find $\varphi^{+}-\varphi^{-}=-E^{+}$.

The more precise argument given in the Appendix shows that $\left(\varphi^{+}-\varphi^{-}\right) /\left|E^{+}\right|$decreases monotonically with $\left|E^{+}\right|$and is bounded as 


$$
\begin{aligned}
& 0 \leq \frac{\varphi^{+}-\varphi^{-}}{\left|E^{+}\right|}-1 \leq \frac{2 b}{\left|E^{+}\right|}+O\left[\left(\frac{b}{\left|E^{+}\right|}\right)^{2} \ln \left(\frac{\left|E^{+}\right|}{b}\right)\right] \\
& \quad \text { for }\left|\mathbf{E}^{+}\right| \rightarrow \infty,
\end{aligned}
$$

where $b>0$ is some constant. The result

$$
\varphi^{+}-\varphi^{-}=-E^{+}+b^{\prime}+O\left(\frac{\ln \left|E^{+}\right|}{E^{+}}\right)
$$

follows. It shows that the first correction to the leading behavior $\varphi^{+}-\varphi^{-} \sim-E^{+}$is just a constant, not a logarithmic term. We thus can choose the gauge of $\varphi$ as $\varphi^{-}+b^{\prime}=0$ to find $\varphi^{+}=-E^{+}+O\left(\ln \left|E^{+}\right| / E^{+}\right)$.

The simplest generalization of this result to curved fronts in strong fields, $\left|E^{+}\right| \gg 1$, suggests the boundary condition

$$
\varphi^{+}=\hat{\mathbf{n}} \cdot(\nabla \varphi)^{+},
$$

replacing the Lozansky-Firsov boundary condition (28). Boundary condition (40) together with the Laplace equation $\boldsymbol{\nabla} \varphi=0$ (1) and the interfacial velocity $\mathbf{v}=\boldsymbol{\nabla} \varphi$ (27) defines our version of the moving boundary model describing the region outside the streamer and the consecutive motion of its boundary.

\section{B. Discussion}

The model formulated here belongs to a class of Laplacian moving boundary models describing a variety of phenomena. In particular, it is intimately related to the extensively studied models of viscous fingering [21,22] and solidification in undercooled melts $[23,24]$. In all these models the boundary separates an interior region from an exterior region, where the relevant field obeys either the Laplace equation or a diffusion equation, and the velocity of the interface is determined by the gradient of this field.

If we replace the boundary condition (40) by $(28), \varphi^{+}$ $=$ const, the model becomes equivalent to a simple model of viscous fingering where surface tension effects are neglected. This "unregularized" model is known to exhibit unphysical cusps within finite time $[28,29]$. To suppress these cusps, in viscous fingering a boundary condition involving the curvature of the interface is used. The physical mechanism for this boundary condition is surface tension. As mentioned in the Introduction, the kinetic undercooling boundary condition (40) is used in the context of solidification. In that case, however, the relevant temperature field obeys the diffusion equation. From a purely mathematical point of view, our model with specific conditions on the outer boundaries far away from the moving interface has been analyzed in [30-32]. It has been shown [32] that with outer boundary conditions appropriate for Hele-Shaw cells, the kinetic undercooling condition selects the same stable Saffman-Taylor finger as curvature regularization. Furthermore, it has been proven $[30,31]$ that an initially smooth interface stays smooth for some finite time. This regularizing property of the boundary condition (40) is also supported by our previous and ongoing work $[18,19,33]$.

In applying an interface model to streamer propagation, an important difference from the other physical systems mentioned above must be noted. For the other systems mentioned the moving boundary model directly results from the macroscopic physics, irrespective of the motion of the boundary: A sharp interface with no internal structure $a$ priori is present. In contrast, a streamer emerges from a smooth seed of electron density placed in a strong electric field, and the screening layer, which is an essential ingredient of the moving boundary model, arises dynamically, with properties determined by the electric field and thus coupled to the velocity of the boundary. The model therefore does not cover the initial "Townsend" or avalanche stage of an electric discharge [11] that is also visible in Fig. 1, but can only be applied to fully developed negative streamers. Furthermore, being explicitly derived for planar fronts, the validity of the boundary condition for more realistic curved streamer fronts must be tested. This issue is discussed in the next section.

\section{CURVED STREAMER FRONTS IN TWO DIMENSIONS}

\section{A. Illustration of numerical results for fully developed streamers}

We solve the PDE model (12)-(14) in two dimensions, using the numerical code described in detail in [13]. (Previous simulation work was in three spatial dimensions assuming radial symmetry of the streamer; the results are very similar.) In the electron current besides the drift term $-\sigma \mathbf{E}$, the diffusive contribution $(D>0)$ is taken into account. This clearly is adequate physically, and on the technical level it smooths the discontinuous shock front. The price to be paid is some ambiguity in defining the position of the moving boundary, see below.

Planar fronts with $D>0$ have been analyzed in $[15,16,25]$, for further discussion and illustrations, we refer to Sec. 2 of [25]. It is found that diffusion enhances the front velocity by a term

$$
v_{n, D}=2 \sqrt{D\left|E^{+}\right| \alpha\left(\left|E^{+}\right|\right)}
$$

that must be added to the velocity $v_{n \text {,drift }}=\left|E^{+}\right|$. Furthermore, diffusion creates a leading edge of the electron density in the forward direction which decreases exponentially on scale

$$
\ell_{D}=\sqrt{\frac{D}{\left|E^{+}\right| \alpha\left(\left|E^{+}\right|\right)}} .
$$

This scale must be compared to the thickness of the screening layer for $D=0, \ell_{\alpha} \sim 1 / \alpha\left(\left|E^{+}\right|\right)$. For large $\left|E^{+}\right|$and small $D$ both the ratios $v_{n, D} / v_{n \text {,drift }}$ and $\ell_{D} / \ell_{\alpha}$ are of order $\sqrt{D /\left|E^{+}\right|}$ $\ll 1$. This, by itself, does not imply that diffusion can be neglected since the term $D \boldsymbol{\nabla} \sigma$ is a singular perturbation of the diffusion-free model. However, in our numerical solutions the main effect of diffusion is found to amount to some rescaling of the parameters in the effective moving boundary model, see below. This is consistent with the observation that for long wavelength perturbations of planar fronts, the limit $D \rightarrow 0$ is smooth [25].

In our numerical calculations, we take $D=0.1$, which is appropriate for nitrogen. For $\alpha(|\mathbf{E}|)$ the Townsend form (9) is used. We start with an electrically neutral, Gaussian shaped 


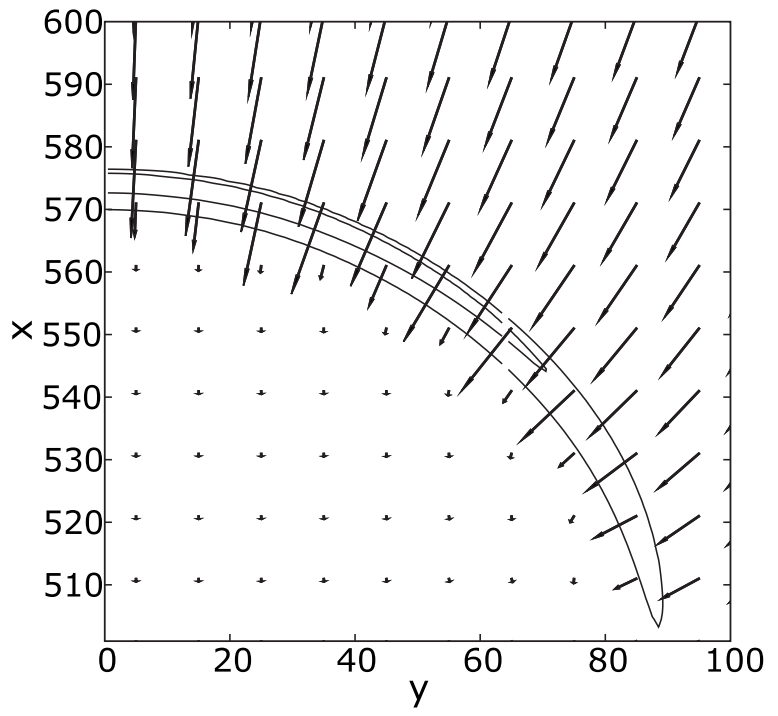

FIG. 3. Zoom into the right half of the symmetric streamer head at time step $t=120$ of Fig. 1 in a background field of $E_{0}=1$. Shown are level lines of negative space charge density $\sigma-\rho$ (at levels $1 / 3$ and $2 / 3$ of the maximal density) and arrows indicating the local electric field at the foot point of the arrow.

ionization seed, placed in a constant external electric field $\mathbf{E}=-E_{0} \hat{\mathbf{x}}$. The total simulation area was $0 \leq x \leq 2048$ and $-1024 \leq y \leq 1024$. We performed runs for $0.5 \leq E_{0} \leq 2$. Since the thickness of the screening layer decreases with increasing $E_{0}$, higher fields need better numerical resolution, enhancing the numerical cost considerably. In view of the results shown below we do not expect qualitative changes for $E_{0}>2$.

The system (12)-(14) is solved numerically with a spatial discretization of finite differences in adaptively refined grids and a second-order explicit Runge-Kutta time integration, as described in detail in [13], with the difference that the method described there was for three-dimensional streamers with cylindrical symmetry and here we adapted it to truly two-dimensional systems. The highest spatial resolution in the area around the streamer head was $\Delta x=\Delta y=1 / 4$ for all simulations.

For external field $E_{0}=1$, Fig. 1 illustrates the temporal evolution of the streamer head. We see that, initially, a screening layer forms out of an ionization avalanche; this process is discussed in detail in [11]. The width of the layer rapidly reaches some almost constant value that depends on $E_{0}$. The head develops into a somewhat flattened semicircle, with the radius increasing with time. This stage of evolution will be addressed as the fully developed streamer. Figure 3 zooms into the streamer head at this stage, showing lines of constant charge density $\rho-\sigma$ together with electric field vectors. Evidently screening is not complete. A small, essentially constant field exists behind the streamer head. This is illustrated in Fig. 4, which shows the variation of the electric field and of the excess charge along the symmetry line $y=0$. This figure shows also that the spatial positions of the maxima of $|\mathbf{E}|$ and of $\sigma-\rho$ do not coincide precisely; in fact, the maximum of $|\mathbf{E}|$ lies within the diffusive leading edge of the front; the small width $\ell_{D}$ of this diffusive layer replaces

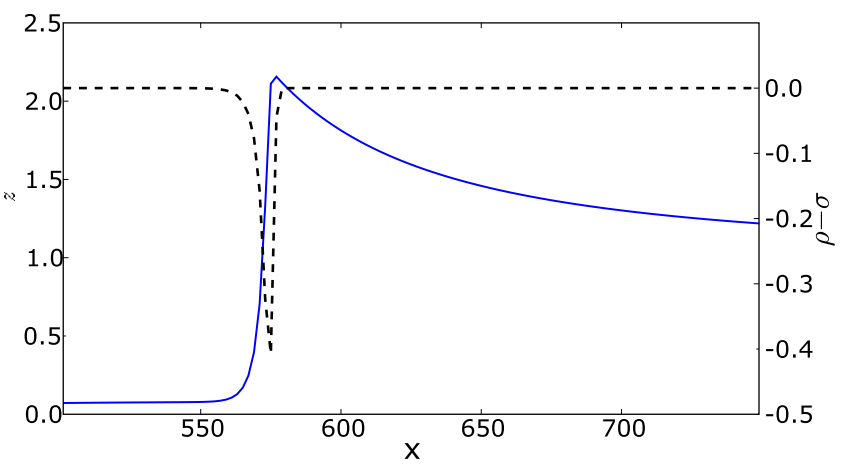

FIG. 4. (Color online) The same case as in Fig. 3, plotted is now the negative electric field (solid line, axis on the left-hand side) and the space charge density (dashed line, axis on the right-hand side) on the axis of the streamer. The plot can be compared to the lower panel in Fig. 2 showing a planar front with vanishing diffusion.

the jump of the electron density for $D=0$. We furthermore observe that the field behind the front is suppressed by about a factor of $1 / 20$ compared to the maximal value $|\mathbf{E}| \approx 2.1$, or equivalently to $\approx E_{0} / 10$. This screening increases with increasing background electric field $E_{0}$ : from $\approx E_{0} / 7$ for $E_{0}$ $=0.5$ to $\approx E_{0} / 20$ for $E_{0}=2.0$. (The maximal field enhancement in these cases is $\gtrsim 2 E_{0}$ in the fully developed streamer briefly before branching.)

\section{B. Test of the assumptions of the moving boundary model}

The moving boundary model is concerned only with the exterior region. Recalling the defining equations

$$
\begin{gathered}
\Delta \varphi=0 \quad \text { in exterior region, } \\
v_{n}=-\hat{\mathbf{n}} \cdot \mathbf{E}^{+}, \\
\varphi^{+}=-\hat{\mathbf{n}} \cdot \mathbf{E}^{+},
\end{gathered}
$$

we note that all explicit reference to the physics in the interior is absent, notwithstanding our derivation in Sec. III. Now the first of these equations evidently holds as soon as the diffusive leading edge of the electron density has a negligible space charge density. Also the second relation holds for any smooth shock front $(D=0)$ of the PDE model. The boundary condition (43), however, was derived only for planar fronts in strong external fields $E_{0} \gg 1$.

To check whether the moving boundary model adequately represents the evolution of curved streamer fronts for small diffusion and for external fields of order unity, we first must choose a precise definition of the interface. As illustrated in Figs. 3 and 4, the screening layer is fairly thin, but nevertheless it involves the two length scales $\ell_{D}$ and $\ell_{\alpha}$ and thus shows some intrinsic structure. We define the moving boundary to be determined by the maximum of $|\mathbf{E}(x, y)|$ along intersections perpendicular to the boundary. In precise mathematical terms a parameter representation $\left[x_{b}(u), y_{b}(u)\right]$ of the boundary obeys the equation 


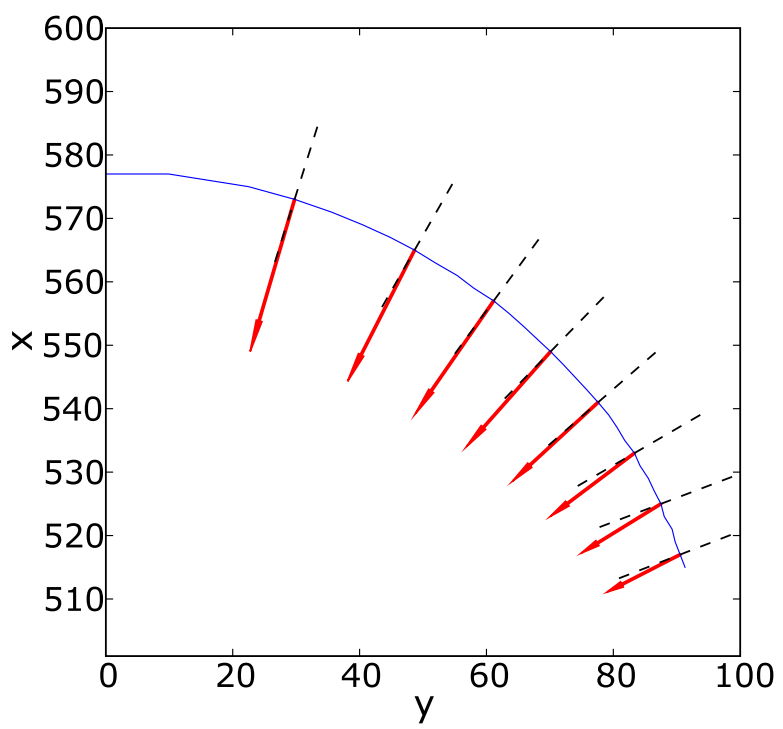

FIG. 5. (Color online) Again the situation of Figs. 3 and 4. Shown is now the boundary defined by the maximum of $E$ on normal intersections (solid line), the local directions of the surface normal (dashed lines), and the local electric fields (solid arrows).

$$
0=\hat{\mathbf{n}}(u) \cdot \boldsymbol{\nabla}|\mathbf{E}|_{x=x_{b}(u), y=y_{b}(u)},
$$

where

$$
\hat{\mathbf{n}}(u) \sim\left(\frac{d y_{b}}{d u},-\frac{d x_{b}}{d u}\right)
$$

is the normal to the boundary at point $\left[x_{b}(u), y_{b}(u)\right]$. To motivate this choice we note that the moving boundary model explicitly refers only to $\mathbf{E}$ and not to the excess charge distribution. In practice we determine $\left[x_{b}(u), y_{b}(u)\right]$ by first searching for the maxima of $|\mathbf{E}|$ along horizontal or vertical intersections, and we then iteratively refine the so determined zero-order approximation. We always follow the boundary up to the point where the local value of $\left|\mathbf{E}^{+}\right|$equals $E_{0}$. This covers all of the head of the streamer, where the essential physics takes place. Figure 5 shows the resulting boundary corresponding to the snapshot of Fig. 3. We observe that the direction of $\mathbf{E}$ is close to, but does not precisely coincide with, the normal direction on the interface (except on the symmetry axis, of course). The boundary is not equipotential but a small component of the electric field tangential to the boundary drives the electrons toward the tip. This effect counteracts the stretching of the space charge layer perpendicular to the direction of streamer motion (see Fig. 1), which in itself would lead to a weakening of screening.

With the so defined interface we have checked that $v_{n}$ depends linearly on $E^{+}$within the numerical precision, therefore Eq. (42) holds, except for an increase of the ratio $v_{n} /\left|\hat{\mathbf{n}} \cdot \mathbf{E}^{+}\right|$, which is an expected effect of diffusion $[15,16,25]$; this effect can be absorbed into a rescaling of time.

The essential test of the boundary condition (43) is shown in Fig. 6. It shows how $\varphi^{+}$depends on $\left|\hat{\mathbf{n}} \cdot \mathbf{E}^{+}\right|$along the boundary for several values of $E_{0}$ measured at times where

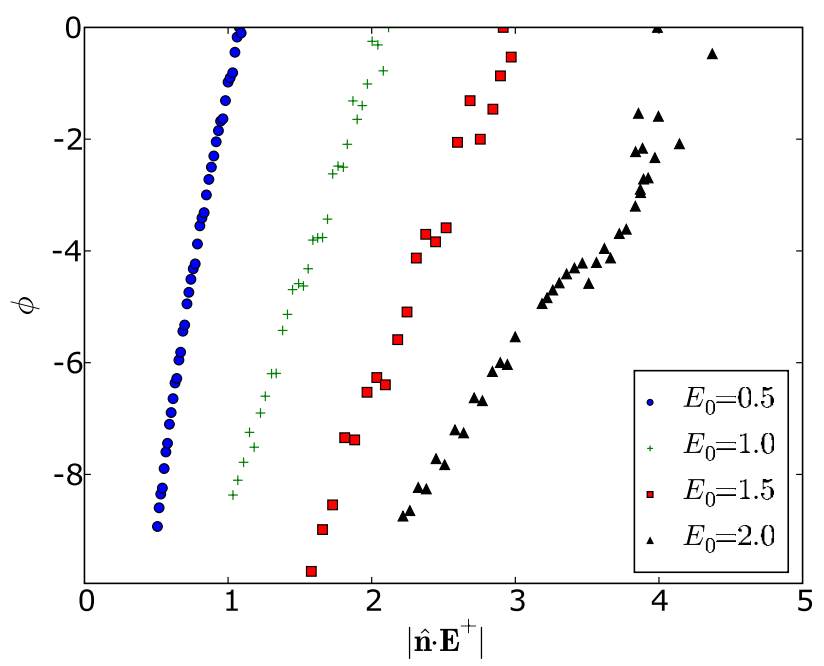

FIG. 6. (Color online) $\varphi^{+}$as a function of $E^{+}$along the interface for different background fields $E_{0}$. A gauge constant $\varphi^{+}\left(E_{\max }^{+}\right)$is subtracted from $\varphi^{+}$for each data set. For each electric field, the data were extracted at times long enough for the space charge layer to be fully developed, but always before the streamer branches. These times were $t=500$ for $E_{0}=0.5, t=120$ for $E_{0}=1.0, t=43.6$ for $E_{0}$ $=1.5$, and $t=35$ for $E_{0}=2.0$.

the streamer is fully developed. For each set of data we first determine $\varphi^{+}$at the maximum of $E^{+}$, i.e., at $y=0$. This constant $\varphi^{+}\left(E_{\max }^{+}\right)$is subtracted from all values $\varphi^{+}$along the interface. Except for the smallest external field $E_{0}=0.5$, the plots in Fig. 6 clearly are linear within the scatter of the data. Even for $E_{0}=0.5$ the curvature is very small. (We note that, with increasing $E_{0}$, the width $\ell_{D}$ of the diffusion layer decreases and approaches the limiting spatial resolution of the numerics [13]. This explains the increasing scatter of the data with increasing $E_{0}$.) Furthermore, as is illustrated in Fig. 7 for $E_{0}=1.0$, for a fixed $E_{0}$ the slope of the relation between $\varphi^{+}$and $\left|\hat{\mathbf{n}} \cdot \mathbf{E}^{+}\right|$does not depend on time. Thus, these numerical results can be summarized by the relation

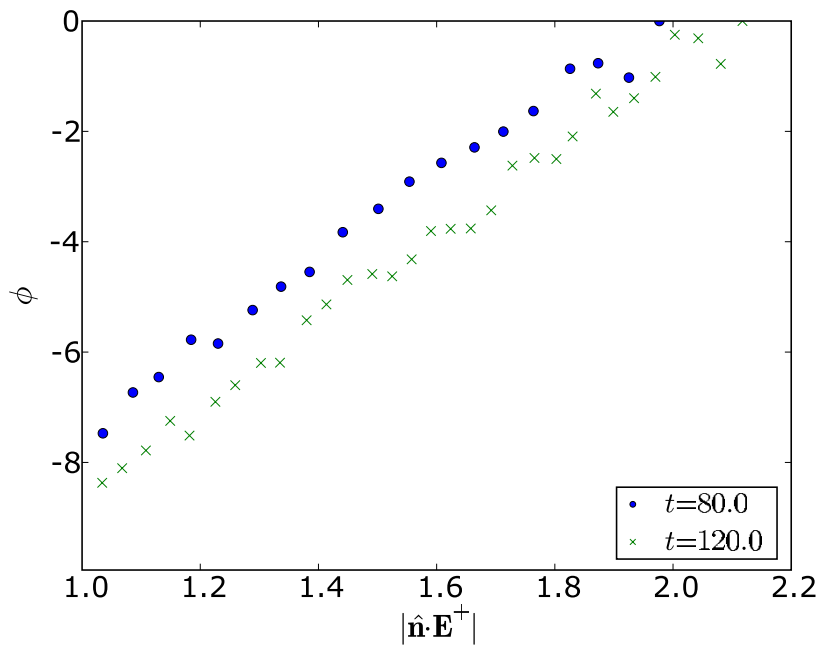

FIG. 7. (Color online) $\varphi^{+}$as a function of $E^{+}$in a background field $E_{0}=1$ for times $t=80$ and 120 . The slope is the same. 


$$
\varphi^{+}=\varphi_{0}\left(E_{0}, t\right)-\ell\left(E_{0}\right) \hat{\mathbf{n}} \cdot \mathbf{E}^{+} .
$$

Of course, neither the PDE model nor the moving boundary approximation depend on the gauge $\varphi_{0}\left(E_{0}, t\right)$, which thus can be ignored. The prefactor $\ell\left(E_{0}\right)$ can be absorbed into the length scale of the moving boundary model, with a compensating change of the time scale to preserve Eq. (42). As mentioned above, this rescaling also can absorb the enhancement of $v_{n}$ due to diffusion. As a result, the model (41)-(43) adequately appears to describe also fully developed curved streamer fronts.

We finally note that the parameter $\ell\left(E_{0}\right)$ decreases with increasing $E_{0}$, and it is well conceivable that for $E_{0} \rightarrow \infty$ it tends to 1 , as predicted by our analysis of planar fronts. Furthermore this behavior parallels the behavior of the thickness of the screening layer, suggesting the very plausible assumption that it is this thickness which sets the spatial scale of the model also away from the limit $E_{0} \rightarrow \infty$.

\section{CONCLUSIONS}

Starting from a PDE model of an anode-directed streamer ionization front, we have derived a boundary condition valid for a moving boundary model of the streamer stage of the discharge. Due to the finite width of the space charge layer surrounding the streamer head, in a moving boundary approximation the electric potential must be discontinuous across the boundary, and the boundary condition (43) proposed here accounts for this jump in a very simple way. Our analytical derivation is restricted to planar fronts in extreme external fields $E_{0} \rightarrow \infty$, but the analysis of numerical solutions of the PDE model shows that the boundary condition also applies to (two-dimensional) curved ionization fronts in weaker external fields. We conclude that the moving boundary model adequately represents the evolution of negative streamer fronts. This conclusion can also be drawn from studies of periodic arrays of interacting streamers that show strong similarities with Saffman-Taylor fingers and are presented elsewhere [20].

As with other moving boundary models in two dimensions, we now are in a position to use powerful conformal mapping techniques to analytically attack questions, such as the stability of streamers against branching. Some first results can be found in Refs. [18,19,33].

The moving boundary model does not explicitly refer to the interior of the streamer and thus leaves open questions concerning the role of the residual electric field and the resulting currents inside the streamer. Analyzing such questions within the framework of the minimal PDE model should lead to a more detailed understanding of the structure of the space charge layer for curved fronts and should clarify the physics underlying the phenomenological length parameter $\ell\left(E_{0}\right)$ occurring in Eq. (44). This problem, which is important for fully understanding the physics of the streamer, is left for future work.

\section{ACKNOWLEDGMENTS}

Two of the authors (F.B., A.L.) were both supported by The Netherlands Organization for Scientific Research NWO,
F.B. through Contract No. 633.000.401 within the program "Dynamics of Patterns," and A.L. by the Foundation for Technological Sciences STW, Contract No. 06501. One of the authors (B.M.) acknowledges support from CWI.

\section{APPENDIX: BOUNDS ON $\varphi^{+}-\varphi^{-}$}

Basic for our discussions are the properties of $\alpha(\eta)$ quoted in Eq. (15), valid for $\eta \geq 0$,

$$
\begin{gathered}
0 \leq \alpha(\eta) \leq 1, \quad \lim _{\eta \rightarrow \infty} \alpha(\eta)=1, \\
\frac{d \alpha}{d \eta} \geq 0 .
\end{gathered}
$$

We furthermore add the physically reasonable condition that

$$
0<b=\sup _{\eta \geq 0}\left(-\frac{d \alpha(1 / \eta)}{d \eta}\right)<\infty
$$

exists, so that $\alpha(\eta)$ obeys the bound

$$
\alpha(\eta) \geq 1-\frac{b}{\eta} .
$$

We now rewrite Eq. (22) for $\rho(E)$ as

$$
\rho(E)=\left(\left|E^{+}\right|-|E|\right) \bar{\rho}\left(\frac{E}{E^{+}},\left|E^{+}\right|\right),
$$

where

$$
\bar{\rho}\left(z,\left|E^{+}\right|\right)=\int_{0}^{1} \alpha\left\{\left|E^{+}\right|[z+(1-z) y]\right\} d y .
$$

Equation (33) for $\varphi^{+}-\varphi^{-}$takes the form

$$
\frac{\varphi^{+}-\varphi^{-}}{\left|E^{+}\right|}=\int_{0}^{1} \frac{d x}{\bar{\rho}\left(x,\left|E^{+}\right|\right)} .
$$

The assumption (A1) that $\alpha(E) \leq 1$ for all $E$ leads directly to the lower bound

$$
\frac{\varphi^{+}-\varphi^{-}}{\left|E^{+}\right|} \geq 1
$$

We note that a better lower bound can be obtained from the fact that since $\alpha\left\{E^{+}[x(1-y)+y]\right\}$ increases with $x$, the function $\bar{\rho}\left(x, E^{+}\right)$obeys

$$
\bar{\rho}\left(x,\left|E^{+}\right|\right) \leq \alpha\left(\left|E^{+}\right|\right) .
$$

This leads to the improved lower bound

$$
\varphi^{+}-\varphi^{-} \geq \frac{\left|E^{+}\right|}{\alpha\left(\left|E^{+}\right|\right)},
$$

illustrating that weak fields cannot be screened since $\left|E^{+}\right| / \alpha\left(\left|E^{+}\right|\right)$typically diverges for $\left|E^{+}\right| \rightarrow 0$.

To derive an upper bound valid for large fields, we assume $\left|E^{+}\right|>b$ and split the integral in Eq. (A7) as 


$$
\int_{0}^{1} \frac{d x}{\bar{\rho}\left(x,\left|E^{+}\right|\right)}=I_{1}+I_{2},
$$

where

$$
\begin{aligned}
& I_{1}=\int_{0}^{b /\left|E^{+}\right|} \frac{d x}{\bar{\rho}\left(x,\left|E^{+}\right|\right)}, \\
& I_{2}=\int_{b /\left|E^{+}\right|}^{1} \frac{d x}{\bar{\rho}\left(x,\left|E^{+}\right|\right)} .
\end{aligned}
$$

By virtue of Eq. (A2), $\bar{\rho}\left(x,\left|E^{+}\right|\right)$increases with $x$, which immediately yields the bound

$$
I_{1} \leq \frac{b}{\left|E^{+}\right|} \bar{\rho}\left(0,\left|E^{+}\right|\right)^{-1} .
$$

Evaluating $\bar{\rho}\left(0,\left|E^{+}\right|\right)$with the bound (A4) on $\alpha(\eta)$ yields

$$
I_{1} \leq \frac{b}{\left|E^{+}\right|}\left(1-\frac{b}{\left|E^{+}\right|}\left[1+\ln \left(\left|E^{+}\right| / b\right)\right]\right)^{-1}
$$

To evaluate $I_{2}$ we write

$$
\begin{aligned}
\bar{\rho}\left(x,\left|E^{+}\right|\right)= & \int_{0}^{x} \alpha\left\{\left|E^{+}\right|[x+(1-x) y]\right\} d y \\
& +\int_{x}^{1} \alpha\left\{\left|E^{+}\right|[y+(1-y) x]\right\} d y \\
> & \int_{0}^{x} \alpha\left(\left|E^{+}\right| x\right) d y+\int_{x}^{1} \alpha\left(\left|E^{+}\right| y\right) d y
\end{aligned}
$$

$$
>1-\frac{b}{\left|E^{+}\right|}+\frac{b}{\left|E^{+}\right|} \ln x \text {. }
$$

This result yields

$$
\begin{aligned}
I_{2} & <1-\frac{b /\left|E^{+}\right|}{1-b /\left|E^{+}\right|} \int_{b /\left|E^{+}\right|}^{1} \frac{\ln x}{1-b(1-\ln x) /\left|E^{+}\right|} d x \\
& <1-\frac{b /\left|E^{+}\right|}{1-b /\left|E^{+}\right|} \frac{1}{1-b\left[1-\ln \left(b /\left|E^{+}\right|\right)\right] /\left|E^{+}\right|} \int_{b /\left|E^{+}\right|}^{1} \ln x d x \\
& =\frac{1}{1-b /\left|E^{+}\right|} .
\end{aligned}
$$

Collecting all the results (and recalling $b /\left|E^{+}\right|<1$ ), we found in this appendix that

$$
\frac{\varphi^{+}-\varphi^{-}}{\left|E^{+}\right|}<\frac{1}{1-b /\left|E^{+}\right|}+\frac{b /\left|E^{+}\right|}{1-\frac{b}{\left|E^{+}\right|}\left[1+\ln \left(\left|E^{+}\right| / b\right)\right]}
$$

which for large $\left|E^{+}\right| / b$ leads to the bound (38) given in the main text. We note, in particular, that $\varphi^{+}-\varphi^{-}$does not contain a contribution of order $\ln \left(\left|E^{+}\right| / b\right)$, so that the leading (constant) correction to $\varphi^{+}-\varphi^{-}=-E^{+}$can be gauged away.

We finally note that Eqs. (A6) and (A2) imply that $\bar{\rho}\left(x,\left|E^{+}\right|\right)$increases monotonically with $\left|E^{+}\right|$, and thus that $\left(\varphi^{+}-\varphi^{-}\right) /\left|E^{+}\right|$decreases monotonically.
[1] H. Raether, Z. Phys. 112, 464 (1939).

[2] L. B. Loeb and J. M. Meek, The Mechanism of the Electric Spark (Stanford University Press, Stanford, CA, 1941).

[3] Yu. P. Raizer, Gas Discharge Physics (Springer, Berlin, 1991).

[4] U. Ebert et al., Plasma Sources Sci. Technol. 15, S118 (2006).

[5] C. Wu and E. E. Kunhardt, Phys. Rev. A 37, 4396 (1988).

[6] S. K. Dhali and P. F. Williams, Phys. Rev. A 31, 1219 (1985).

[7] S. K. Dhali and P. F. Williams, J. Appl. Phys. 62, 4696 (1987).

[8] P. A. Vitello, B. M. Penetrante, and J. N. Bardsley, Phys. Rev. E 49, 5574 (1994).

[9] M. Arrayás, U. Ebert, and W. Hundsdorfer, Phys. Rev. Lett. 88, 174502 (2002).

[10] A. Rocco, U. Ebert, and W. Hundsdorfer, Phys. Rev. E 66, 035102(R) (2002).

[11] C. Montijn and U. Ebert, J. Phys. D 39, 2979 (2006).

[12] C. Montijn, U. Ebert, and W. Hundsdorfer, Phys. Rev. E 73, 065401(R) (2006).

[13] C. Montijn, W. Hundsdorfer, and U. Ebert, J. Comput. Phys. 219, 801 (2006).

[14] E. D. Lozansky and O. B. Firsov, J. Phys. D 6, 976 (1973).

[15] U. Ebert, W. van Saarloos, and C. Caroli, Phys. Rev. Lett. 77, 4178 (1996).

[16] U. Ebert, W. van Saarloos, and C. Caroli, Phys. Rev. E 55,
1530 (1997).

[17] B. Meulenbroek, A. Rocco, and U. Ebert, Phys. Rev. E 69, 067402 (2004).

[18] B. Meulenbroek, U. Ebert, and L. Schäfer, Phys. Rev. Lett. 95, 195004 (2005).

[19] U. Ebert, B. Meulenbroek, and L. Schäfer, SIAM J. Appl. Math. 68, 292 (2007).

[20] A. Luque, F. Brau, and U. Ebert, e-print arXiv:0708.1722.

[21] P. G. Saffman and G. I. Taylor, Proc. R. Soc. London, Ser. A 245, 317 (1958).

[22] D. Bensimon, L. P. Kadanoff, S. Liang, B. I. Shraiman, and C. Tang, Rev. Mod. Phys. 58, 977 (1986).

[23] Y. Saito, Statistical Physics of Crystal Growth (World Scientific, New Jersey, 1996).

[24] Y. Pomeau and M. Ben Amar, in Solids Far from Equilibrium, edited by C. Godréche (Cambridge University Press, Cambridge, 1992).

[25] G. Derks, U. Ebert, and B. Meulenbroek, e-print arXiv:0706.2088.

[26] C. Li, W. J. M. Brok, U. Ebert, and J. J. A. M. van der Mullen, J. Appl. Phys. 101, 123305 (2007).

[27] M. Arrayás and U. Ebert, Phys. Rev. E 69, 036214 (2004).

[28] B. Shraiman and D. Bensimon, Phys. Rev. A 30, 2840 (1984). 
[29] S. D. Howison, J. R. Ockendon, and A. A. Lacey, Q. J. Mech. Appl. Math. 38, 343 (1985).

[30] Yu. E. Hohlov and M. Reissig, Eur. J. Appl. Math. 6, 421 (1995).
[31] G. Prokert, Eur. J. Appl. Math. 10, 607 (1999).

[32] S. J. Chapman and J. R. King, J. Eng. Math. 46, 1 (2003).

[33] S. Tanveer, U. Ebert, F. Brau, and L. Schäfer (unpublished). 